\title{
Design and Implementation of Database Security Based on Server SQL Online Course Selection
}

\author{
Jingxiu $\mathrm{Xu}^{1, \mathrm{a}}$ and Jinmeng Xiong ${ }^{2, \mathrm{~b}}$ \\ ${ }^{1}$ Huanggang Normal University, Huangzhou two, Xingang District, Huanggang City, Hubei Province, \\ China \\ ${ }^{2}$ Wisdri Engineering \& Research Incorporation Limited No.33, Daxue Yuan Ave, East Lake \\ High-Tech Development Zone, Wuhan, Hubei Province, P.R.China \\ aXujingxiu123@126.com, bameng821226@163.com
}

Keywords: Database; Security; Design

\begin{abstract}
With the rapid development of computer and the popularization of computer network, the application of database system has gradually become the core part of the information management system, which is used in all aspects: the country, the enterprise, the commercial and daily life. To national government departments, the defense industry, science and technology development areas, A large part of the traditional database is used in the business field, such as the securities industry, the bank, the sales department, the hospital, the school, the company or the enterprise unit, now a small supermarket is also managed by the database data. The security of the database is even more important. This paper discusses the factors that threaten the security of the database, mainly describes the security design and implementation of the online course selection database based on Server SQL, so as to achieve the requirements of database security design.
\end{abstract}

\section{Introduction}

With the rapid development of the computer and the Internet, the application of computer technology has from the science research department extended to all walks of life, data processing has become the main aspects of the computer application, due to the existence and influence of various technical and non-technical factors, the data is growing rapidly, the data security of accessing, storing and managing the information is extremely important [9]. To prevent the illegal use of the company's data which may cause data leakage, change or damage, resulting in unnecessary losses.

There are several main factors that threaten the security of the database.

Non Authorized Users of Malicious Access and Destruction to the Database. Some hackers and criminals hunt for user name and password when the user access to the database, and masquerade as a legal user to steal, modify or even destroy the user data. Therefore, it is necessary to prevent the illegal operation of the database security, to ensure that data is protected from unauthorized access and damage, the security measures provided by the database management system mainly include: user authentication, access control and view technology [11].

Important or Sensitive Data in the Database Is Compromised. Hackers and hostile elements do everything possible to steal important data in the database, some of the confidential information is exposed. In order to prevent data leakage, the main technology provided by the database management system is: mandatory access control, data encryption, storage and encryption transmission, etc.

The following is my design online elective database security based on the Server SQL, its design and implementation process is shown below:

One, Legitimate Login Account Login Database System, Unauthorized Entry into the System Is Prohibited

User identity authentication is the most protective measure for the security of database management 
system. Each user has a user ID in the system. Inside the system records all legitimate users of the identity, system identification is defined by the system to provide a certain way to allow the user to identify their own name or identity. Each time the user requests to enter the system, checked by the system, provide authority to access to database management systems after identification [10]

Legitimate login required through the Windows authentication and Server SQL authentication dual authentication mode login, Unauthorized entry into the system without the creation of a super administrator, The following Fig. 1-1 is the process that login account logliu which online course selection system super administrator created to be through the Server SQL authentication login system.

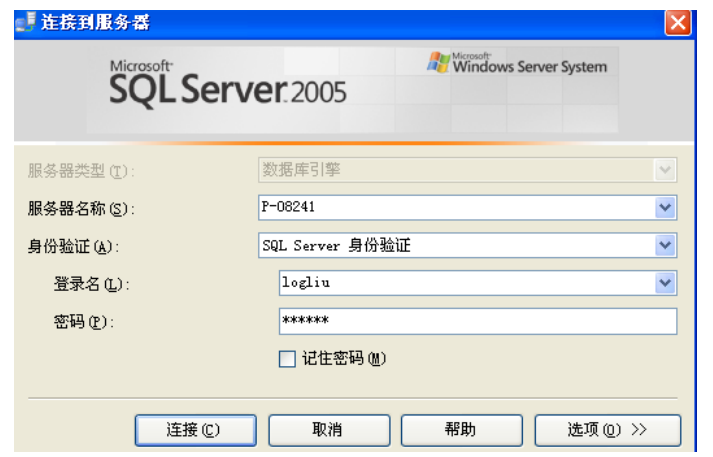

Figure 1. Login account logliu login system

With a login account, but also let logliu become online course database users can connect to the database, that is, to become an online course database users. Hypothesis to logliu the database user name set for the dbuserliu need the super administrator login system, using a system stored procedure code writing as follows: the execution results as shown in Fig. 1-2, in online course data security users have database user dbuserliu.

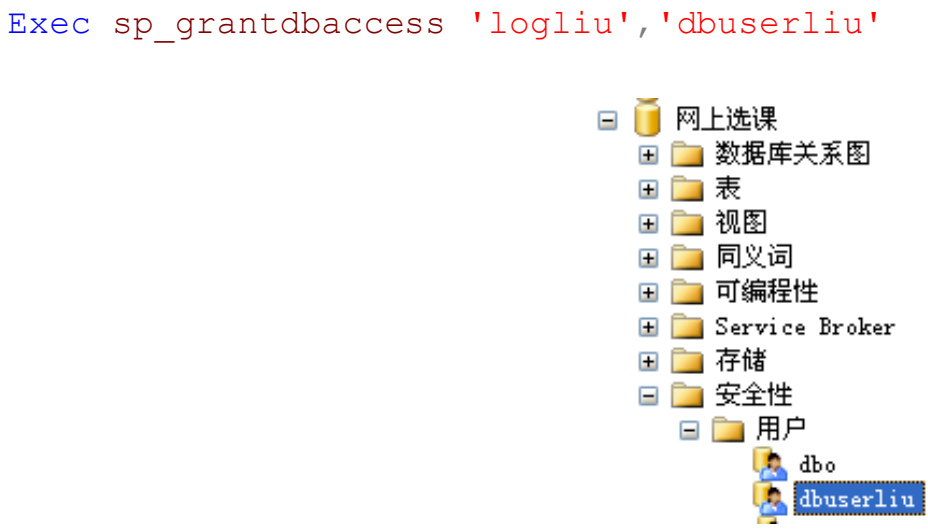

Figure 2. Database user dbuserliu

Through the super administrator to create a login account logliu and setting the database user dbuserliu for the account logliu, the database to a certain extent, to ensure the security of database login and database connection.

\section{Two, Online Course Selection Database Independent Access Control}

Authorized Dbuserliu Access to the Curriculum Schedule Course. The most important thing of database security is to ensure that only authorized users to access the database permissions, while all unauthorized personnel cannot access the data. For example, the students can only authorization to check their own course result, cannot see the other students' course result, but also cannot modify their own course results. This is achieved through the access control mechanism of the database system [12]. The following code is the online course database user dbuserliu authorization to access 
the course schedule course through the super administrator.

Grant select on course to dbuserliu Authorized by this code, database users dbuserliu can only see web course database tables course and other data tables are not entitled to access, in a large extent to ensure the privacy and security of the online course database and other data.

Thus, database discretionary access control policy management is, to a database user by "rights management" command set users accessible object, such as tables, views, stored procedures, and select, insert, update, delete, etc. specific permission to operate, even set for further data table fields select, update permissions.

View Mechanism. Data in the database is stored in a table, and the view is just a result set of one or more tables in accordance with a combination of conditions. the main difference between the table and the view is as follows [3]:

a, the view is already compiled SQL statement. And the tables have actual physical records.

$\mathrm{b}$, the table is the content, the view is just a window to display data in the database table, there is no actual physical record.

c, Tables only use the physical space and the view does not occupy the physical space, the view is only the existence of the concept of logic, the table can be modified in a timely manner, but the view can only be created to modify the statement [8]

$\mathrm{d}$, the table is the inside model, the view is the outside mode [2]

$\mathrm{e}$, view is a way to view the data table, you can query the data that some fields constituted in data table, which just some of the SQL statements. From a security point of view, the view cannot give the user access to the data table, and thus do not know the table structure [4].

$\mathrm{f}$, the table is a table in the global mode, is a real table; view belongs to the local mode table is virtual table [5].

$\mathrm{g}$, the establishment and delete of the view only affect the view itself, does not affect the corresponding basic table.

The Connection of tables and views are shown in: view is a table built on the basic table, its structure (that is, the column) and content (that is, all data lines) are derived from the basic table, which is based on the existence of the basic table. A view can be corresponding to a basic table, you can also correspond to a number of basic tables. View is an abstraction of the basic table and a new relationship established in the logical sense [6], once the table is deleted, the view does not exist.

Access control for different user to define different views, the data object is limited in a certain range, The administrator hides the data to be kept secret by the view mechanism. To provide a certain degree of security protection to the data. The view mechanism indirectly implements the user rights definition of support access predicates [7], but view mechanism main function is to provide the independence of data, the safety protection function is often far from reach the requirements of the application system. Therefore, in practical applications, the view mechanism is usually used in conjunction with the authorization mechanism, first, the view mechanism is used to screen out a part of the secret data, and then define the access rights on the view. As shown in the following Fig. 2-1, this is a good illustration of the advantages of the view.

Combined with the online course database security, create a view v1, request to create a class of 01 software engineering class information view, and access to the view V1 authorization to the database user dbuserliu.

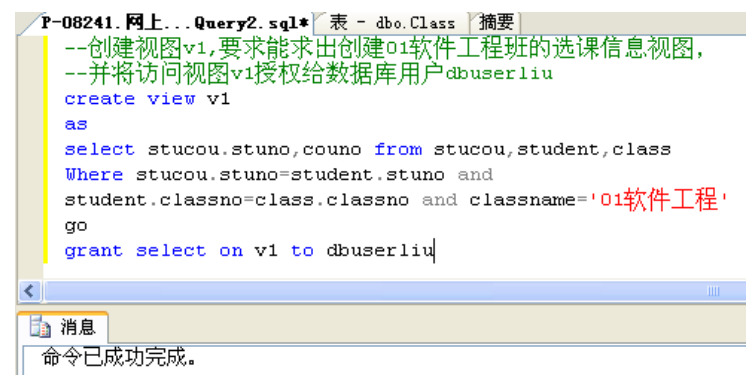

Figure 3. View V1 database user dbuserliu authorization 
Through the operation of Fig. 2-1, super administrator realized create view V1, and through authorized access view V1 to users of the database dbuserliu, and reflected the view function as a virtual table, very good to protect the database base table stucou, student and class data security.

\section{Three, Data Encryption}

In addition to the above mentioned security measures, but also highly sensitive data using data encryption technology. Data encryption is a effective means to prevent confidential data in the database in the storage and transmission [10]. The basic idea of encryption is to transform the original data into a format that cannot be directly identified by a certain algorithm, which makes it impossible for people who don't know the decryption algorithm to know the contents of the data.

Concluding remarks:

This paper is mainly based on SQL server online course database, create the login account logliu and database users dbuserliu, set access to table course permissions for dbuserliu, create a view v1 and grant it access to dbuserliu. So achieve the Server SQL online course database security, and achieve the desired results. Server SQL database security technology also has other methods, such as data encryption, classification control. In the information age, it is worth to study and practice the security of the database for storing and managing the information [1].

\section{References}

[1] Zhang Runlian, Zhang Xiang-li. Case teching method and its application [J]. Journal of Guilin University of Electronic Technology, 2004(4): 102-105. (in Chinese)

[2] Dong Chen, Zhang Ye. Researches on database course design for 'Excellency Plan [J]. Computer Education, 2013(23), 120-123.(in Chinese)

[3] Siberchatz A, Korht H F, Sudarshan S, Database system concepts (concise version) [M].Beijing: China Machine Press,2013.

[4] Yujiang Zhang flipped classroom change. China II] information technology education, 2010 (10).

[5] Liu Chuanbo. SQL Server database security strategy design and application of [J]. business culture (Academic Version of) 2008

[6] He Hanhua.SQL Server database security analysis and application of [J]. software:.2009

[7] Xing et al. Security issues on the SQL Server database on [J]. technology.2008

[8] Du trillion will be such as. SQL Server database management and development tutorials and training.Social.2006

[9] Sun Fengdong. 11g Oracle database basic course [M]. Beijing: Publishing House of electronics industry, 2014

[10] Wang Shan, watermarking. Introduction to database systems (Fifth Edition) [M]. Beijing: Higher Education Press, 2014

[11] Zhang Runlian, Zhang Xiangli, Ye Jin. Case teaching method and its application [J] Journal of Guilin University of Electronic Technology, 2004 (4):102-105

[12] Ceng Jianhua, Xu Renfeng. Server2008 SQL database and its application [M]. Beijing: Higher Education Press, 2007 